\title{
Improving the Productivity and Nutritional Status of Washington Navel Orange Tress by Using Some Nano Compounds and Natural Extracts under Different Irrigation System \\ 1- Vegetative growth and nutritional status
}

Atawia, A. R*.;Abd El-Latif, F. M.* ; El-Badawy, H. E.*;Farag,A. A.** and EL Atrouny, R. A.* * Hort. Dep., Fac. of Agric., Benha Univ. ** Agric. Eng. Dep., Fac. of Agric., Benha Univ. Corresponding Author: abousrie.ahmad@ fagr.bu.edu.eg

\begin{abstract}
The present investigation was undertaken throughout the two successive seasons of 2016 and 2017 at fruit orchard, Faculty of Agriculture at Moshtohor, Benha University, Toukh region, Qalyubeia Governorate, Egypt.

The main goal from study evaluate the effect of some stimulating substances i.e., (yeast extract and nano fertilizer) at different concentrations and irrigation levels on some vegetative growth measurements and leaf nutritional status of Washington navel orange trees budded on sour orange rootstock. Results indicated that, all investigated stimulating substances treatments under study as foliar spray at various concentrations and irrigation with high level resulted in a positive and significant increase in most vegetative growth measurements and improving leaf nutrient contents of Washington navel orange trees in comparison with the control during both seasons of study. The best results were obtained from sprayed trees with yeast extract at 150 $\mathrm{ml} /$ liter+lithovit at $4.0 \mathrm{~g} / \mathrm{liter}$ and irrigation with $3894.1 \mathrm{~m}^{3}$ water / feddan / year compared to the water spray and irrigation with $3127.8 \mathrm{~m}^{3}$ water /feddan/year during both seasons of study.
\end{abstract}

Keywords: Citrus, yeast extract, lithovit, irrigation, vegetative growth and nutritional status.

\section{Introduction}

Citrus is considered one of the most important fruit crops grown in many tropical and subtropical countries. In Egypt, citrus has a great attention and widely cultivated due to importance for local consumption (high nutritive value) and economic importance however, represent a main source for foreign currencies by exportation to many countries of world especially both European and Asian countries. Moreover, it ranks the first in Egyptian fruit production and the second after the grapes in the world as fruit production. The Egyptian Agriculture statistics in 2017 indicate that, citrus total planted area reached 477510 feddans and total citrus production of this area equal 4451644 tons of fruits, this represents $28.78 \%$ and $37.08 \%$ of total fruits orchards area and fruits production, respectively. Total orange cultivated area represents 326484 feddans with total fruits production of 3147545 tons. Ministry of Agriculture, Economic Affairs Sector, 2017.

Yeast is one of the richest natural source of high quality protein, namely the essential amino acids as lysine, tryptophan etc., contains the essential elements and trace nutrients as $\mathrm{Ca}, \mathrm{Fe}$ and $\mathrm{Co}$ etc. and the best sources of vitamins $\left(\mathrm{B}_{1}, \mathrm{~B}_{2}, \mathrm{~B}_{6}\right.$ and $\left.\mathrm{B}_{12}\right)$, also the yeast extract is a valuable source of bioconstituents especially cytokinins(Amer, 2004). Furthermore, many researchers reported that spraying some fruit trees including citrus trees with different stimulating substances such as active dry yeast extract at the different concentrations can promote plant growth may be due to activate root cells at the same time stimulating biosynthesis of endogenous cytokinins from roots, enhancing leaf water status, shoot growth and root pull strength (Demiret al., 2004), stimulation the uptake of $\mathrm{N}, \mathrm{P}$, $\mathrm{K}, \mathrm{Mg}, \mathrm{Ca}, \mathrm{Fe}, \mathrm{Zn}$ and $\mathrm{Cu}$ by the plants (Nelson and Van-Staden, 1984) and stimulation the biosynthesis of chlorophylls, carotenoidsand ascorbic acid which protect photosynthesis apparatus of horticulture plants (Crouch and Van-Staden, 1993) as well as stimulation stem elongation and regulation cell membrane components under drought stress (Smirnoff, 1995 and Fletcher et al., 1988).

Nanotechnology is a promising field of interdisciplinary research. It opens up a wide array of opportunities in various fields like agriculture. The potential uses and benefits of nanotechnology are enormous. Nanoparticles generate both positive and negative biological effects in living cell (Nelet al., 2006). There is increasing amount of research on the biological effects of nanoparticles on higher plants. Similarly, mixture of nanoscales hastened germination in soya bean (Lu et al., 2002).

Nano-fertilizers are used recently as an alternative to conventional fertilizers for slow release and efficient use by plants. Lithovit is recommended by European Community for organic farming according to EWG 2092/91 (Bilal, 2010). It is a natural $\mathrm{CO}_{2}$ foliar fertilizer made from limestone deposits by tribodynamic activation and micronization to levels of 10-20 microns. Most of Lithovit particles remain as thin layer on the surface of leaves and penetrate frequently when they get wet by dew at night. Lithovit could be used for enhancing yield, quality and storage properties of crop especially when plants are subjected to stress, where it acts as a long term reservoir supplying plants with $\mathrm{CO}_{2}$ (Bilal,2010 and Kumar, 2011). 
Lithovit compound containing calcium carbonate (80\%) Magnesium carbonate $(4.6 \%)$ and $\mathrm{Fe}(0.75 \%)$ particles. The beneficial effect of this compound is being contains calcium carbonate $\left(\mathrm{CaCo}_{3}\right)$ decomposes to calcium oxide (Cao) and carbon dioxide $\left(\mathrm{Co}_{2}\right)$ in leaves stomata, and this $\mathrm{Co}_{2}$ increases photosynthesis intensity, leading to increased carbon uptake and assimilation, thereby increasing plant growth ( Carmen et al., 2014 ). Magnesium which is the control elements in chlorophyll molecule and it also as enzyme activator and constituent of many enzymes. Also, Iron is very important for chlorophyll formation and photosynthesis and activities many enzyme system and respiration of plants (Marschner, 1995 and Nadiet al., 2013).

The positive effect of lithovit compound on plant growth and biochemical constituents was reported by ( Abo-Sederaet al., 2015 ) found that, foliar application of lithovit at $3 \mathrm{~g} / \mathrm{l}$ and seaweed extracts increased vegetative growth, total green pods yield and it compounds as well as pod quality of snap bean plants, Also, ( Abd El Ghafaret al., 2016 ) showed that, foliar application with lithovit $0.5 \mathrm{~g} / \mathrm{l}$ significant increased number of umbel, height of number scape , diameter of umbel, chlorophyll and carotenoids, seed yield /plot and seed germination \% of onion plants.

Water is fast becoming an economical score resource in many areas of the world especially in arid and semiarid regions. In Egypt, water is considered as a limited resource because of increasing population. Moreover, water is one of the most important components in biological systems (Salisbury and Ross, 1985). Maximizing the use of irrigation water is essential for increasing of irrigation water demands (Brown, 1999).

Wright and Stark (1990) revealed that, plant growth and development retarded when water supply as restricted. But, Ismail et al., (2007) on pear andKandil and EI-Feky (2006) on apricot used 40, 60,70 or $80 \%$ field capacity (F.C.) and obtained the best growth parameters and yield components with $80 \%$ F.C. Moreover, Cathoun (1975) found that, the increase in tension from zero to 0.33 bar released more than $75 \%$ of water in light textured soil but less than $50 \%$ in heavy ones. Levin et al., (1980), stated that, root distribution depended upon the volume of wetted soil, which was related to soil hydraulic conductivity, the rate and duration of water application. Therefore, using water soil potential at $100-200 \mathrm{mbar}\left(12.94 \mathrm{~m}^{3} /\right.$ tree/year) was recommended as the best level for "Canino" apricot trees in sandy soil (Kandil and EI-Feky, 2006).

This present investigation aimed to study the effect of different some stimulating substances i.e. active dry yeast extract and lithovit at different concentrations as foliar spray on some vegetative growth measurements and leaf nutritional status of bearing Washington navel orange trees under different irrigation levels.

\section{Materials and Methods}

The present dissertation was conducted on fruitful trees of sweet orange "Citrus sinenses L." Belonging to Washington navel orange cultivar during two successive experimental seasons (2016 and 2017).

In this experiment fifty eight-year old Washington navel orange trees budded on sour orange "Citrus aurantium" rootstock and grown in clayloamy soil at Experimental Station of Faculty of Agriculture,Benha University at Moshtohor ,Toukh region, Kaliobia Governorate were the plant material used in this regard to investigate the influence of two irrigation levels and different some stimulating substances i.e. active dry yeast extract and lithovit at different concentrations as foliar spray on some vegetative growth measurements and nutritional status of bearing Washington navel orange trees. Each nutrient compound was investigated solelyor combined to other and foliar spray on vegetative growth.

The experiment was arranged in a factorial designed experiment contained 2 irrigation levels $\left(3894.1 \mathrm{~m}^{3}\right.$ water / feddan / year or $3127.8 \mathrm{~m}^{3}$ water /feddan/year) X 8 stimulating substances $=16$ treatments. Each treatment had three replicates and one tree for each, (randomize complete block design). The sixteen treatments represented the different possible combinations between the following two investigated factors. Trees irrigated with level 1 (3894.1 $\mathrm{m}^{3}$ water / feddan / year) treated with:

1-Control (water spray of trees subjected to only the $\mathrm{N}, \mathrm{P}, \mathrm{K}$ fertilizers programs adopted in the farm).

2-Foliar spray with yeast extract at 150 $\mathrm{ml} / \mathrm{liter}$.

3-Foliar spray with yeast extract at 200 $\mathrm{ml} / \mathrm{liter}$.

4-Foliar spray with lithovit at $2.0 \mathrm{~g} / \mathrm{liter}$.

5 -Foliar spray with lithovit at $4.0 \mathrm{~g} / \mathrm{liter}$.

6-Foliar spray with yeast extract at 150 $\mathrm{ml} / \mathrm{liter}+$ lithovit at $2.0 \mathrm{~g} / \mathrm{liter}$.

7-Foliarspraywithyeast extract at 150 $\mathrm{ml} / \mathrm{liter}+$ lithovit at $4.0 \mathrm{~g} / \mathrm{liter}$.

8-Foliarspraywithyeast extract at 200 $\mathrm{ml} / \mathrm{liter}+$ lithovit at $4.0 \mathrm{~g} / \mathrm{liter}$.

Trees irrigated with level $2\left(3127.8 \mathrm{~m}^{3}\right.$ water /feddan/year) treated with same treatments.

- Yeast extract andlithovit treatments were applied three times in last week of March (full bloom), last week of April and last week of June.

- Level of irrigation water was determined with Cutthroat Flume after Fareg (2007).

\section{Experiment layout:}

Onlate March 2016 and 2017 fourmain branches (limbs/scaffolds) well distributed around each tree 
peripherywerecarefullyselectedandtaggedd uring $1^{\underline{\text { st }}}$ and $2^{\frac{\text { nd }}{}}$ seasons, respectively. Moreover, 20 newlyspringdevelopedshoots werealsolabeled.

1. Vegetative growth measurements:

Onmid October 2017and 2018 years the following vegetative growth parameters were determined.

In this regard, average number of newly developed shoot speronemeter of every tagged limb,average (length\&thickness)and numberofleaves,pereach

labeledshootwereestimated.

\section{Leaf mineralcomposition:}

Representativesamplesoffourthandfifthl eavesfromthebase of spring shoots were collectedfrom each replicate in October during both seasons. Thesampleswerethoroughlywashedwithtap water,rinsedtwicewithdistilledwaterandove ndriedat $80^{\circ} \mathrm{Ctillaconstantweightandfinelyg}$ roundfor determinationof:

a.Total Nitrogen: Total leaf $(\mathrm{N})$ was determined by the modified micro Keldahlmethodmentioned by (Pregl, 1945).

b.Total phosphorus: Totalleaf(P) wasdeterminedbywet materialsafterthemethodsdescribedby usingsulphoricandperichloricacidwhichhasbeenstron glyrecommended by(Piper,1958).
c. Total potassium:
Total leaf $(\mathrm{K})$ was

d.CalciumandMgpercentageaswellasIron,

ManganeseandZincweredeterminedusingthe Atomicab sorption spectrophotometer"PerkinElmer3300"afterChapmanand Pratt(1975).

\section{Statisticalanalysis:}

Alldataobtainedduringbothseasonsweresubjected to analysis ofvarianceaccordingtoSnedecor and Cochran, 1977. Inaddition, significant differences among means weredifferentiated accordingtothe Duncan, multipletestrange(Duncan,1955).

\section{Results and Dissections}

\section{Vegetative growth:}

The response of fruitful Washington navel orange trees to some bio and nano fertilizers (foliar spray) and two irrigation levels was studied through the determination of some vegetative growth parameters (No. of shoots, shoot length, shoot diameter, number of leaves/shoot, leaves fresh weight and leaves dry weight).

\section{A. Specific effect:}

Regarding the specific effect of the fertilizers substances(yeast extract and lithovit) on no. of shoots, shoot length, shoot diameter, number of leaves/shoot, leaves fresh weight and leaves dry weight of Washington navel orange trees data in
Tables (1-6) revealed that, the highest significant values of all measurements were obtained when the trees where sprayed with yeast extract at $150 \mathrm{ml} / \mathrm{liter}$ + lithovit at $4.0 \mathrm{~g} /$ liter.Meanwhile the latest values were obtained when the trees where sprayed with water (control).

Concerning the specific effect of irrigation levels, it was clear that, the highlevel $\left(3894.1 \mathrm{~m}^{3}\right.$ water / feddan / year)was better than the low level (3127.8 $\mathrm{m}^{3}$ water/feddan/years) in enhancing all vegetative growth measurements during two experimental seasons.

\section{B. Interaction effect:}

With referring to the interaction between fertilizers substances and irrigation levels on no. of shoots, shoot length, shoot diameter, number of leaves/shoot, leaves fresh weight and leaves dry weight).

Data in Tables (1-6) indicated that, the maximum values were detected with the combination between foliarspraywithyeast extract at $150 \mathrm{ml} / \mathrm{liter+lithovit}$ at $4.0 \mathrm{~g} / \mathrm{liter}$ and irrigation with $3894.1 \mathrm{~m}^{3}$ water / feddan / yearduring two seasons.

On the other hand, the least values of no. of shoots, shoot length, shoot diameter, number of leaves/shoot, leaves fresh weight and leaves dry weight were observed when the Washington navel orange trees treated with water and irrigated with $3127.8 \mathrm{~m}^{3}$ water /feddan/year during two experimental seasons.

Our results regarding the impact of yeast extract are in general agreed with the findings of El-Shazly.

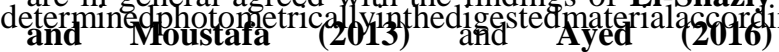
onWashington navel orange, they reported that yeast extract increased growth measurements.

The obtained results concerning the impact of lithovit are confirmed by the findings of Abd El-Aal and Rania - Eid (2018) on soybean plants reported that, foliar application with lithovit caused significant increases in all vegetative growth characteristics as compared with the control treatment. Lithovit compound consists of calcium carbonate (80\%) Magnesium carbonate (4.6\%) and $\mathrm{Fe}(0.75 \%)$ particles. The beneficial effect of this compound leading to increasing carbon uptake and assimilation, thereby increasing plant growth (Carmen et al., 2014).

These results concerning to irrigation levels are in general agreed with the findings of Sanchez et al., (1989) on Verna lemons; Zekri (1991) on citrus rootstocks and Saeed et al., (2005) on jojoba seedlings obvious that, stem length, no. of branches, number of leaves per plant and root length were significantly increased as available water increased.

\section{Leaf mineral content:}

\section{A- Specific effect.}

Regarding leaf mineral contentof Washington navel orange trees as affected by each individual investigated factor (fertilization substances and 
irrigation levels) data in Tables (7-14) display that, the highest value of leaf mineral content was achieved where the trees were sprayed with yeast extract at $150 \mathrm{ml} / \mathrm{liter}+$ lithovit at $4.0 \mathrm{~g} / \mathrm{liter}$ or yeast extract at $150 \mathrm{ml} / \mathrm{liter}+$ lithovit at $2.0 \mathrm{~g} / \mathrm{liter}$.

With respect to the specific effect of irrigation levels, data indicated that, Washington navel orange trees irrigated with $3894.1 \mathrm{~m}^{3}$ water / feddan / year increased leaf mineral content during both seasons of study.

B-Interaction effect:

With respect to the interaction effect between fertilization substancesand irrigation levels on leaf mineral content, data inTables (7-14) display that, the highest value of leaf mineral content was detected with those sprayed trees with yeast extract at $150 \mathrm{ml} /$ liter + lithovit at $4.0 \mathrm{~g} / \mathrm{liter}$ combined with irrigation with $3894.1 \mathrm{~m}^{3}$ water / feddan / year.

On the other hand, the least value of leaf mineral content were recorded with untreated trees (water spray) and irrigated with $3127.8 \mathrm{~m}^{3}$ water /feddan/year during two seasons.
These results are in general agreed with the findings of Badawy-Sabah (2005) on Baladymandarin;Bakry (2007) on Jafa orange trees; Ahmed $\boldsymbol{e t}$ al., (2013) on Washington Navel orange, they reported that sprays trees with yeast extract increased leaf mineral contents i.e. nitrogen, phosphorus, potassium, calcium, magnesium, manganese, iron and zinc.

The present results, concerning the effect of lithovit are in harmony with those reported byAbd El-Aal and Rania - Eid (2018) on soya beanandGhatas and Mohamed (2018) on Cymbopogoncitratus indicated that the application of lithovit gave the highest values leaf chemical compositions (N, P, K, Ca, Mg \% and Fe ppm).

The present results regarding the response of nutritional statusare supported by the early findings of Youssef (1990)oncitrus rootstocksand Maurer et al., (1995) on grapefruit trees, theyfound that, nitrogen, potassiumand phosphorus were increased as available soil water of irrigation increased.

Table 1.Response of No. of shoots / one meter limb of fruitfulWashington navel orange trees to some bio and nano fertilizers (foliar spray) and two irrigation levels during 2016\&2017 experimental seasons.

\begin{tabular}{|c|c|c|c|c|c|c|}
\hline \multirow{2}{*}{ Treatments } & \multicolumn{6}{|c|}{ No. of shoots / one meter limb } \\
\hline & Irrigation1 & Irrigation2 & Mean & Irrigation1 & Irrigation 2 & Mean \\
\hline & \multicolumn{3}{|c|}{ First season } & \multicolumn{3}{|c|}{ Second season } \\
\hline 1 & $14.33 \mathrm{de}$ & $11.67 \mathrm{e}$ & $13.00 \mathrm{C}$ & $19.50 \mathrm{bc}$ & $19.30 \mathrm{bc}$ & $19.40 \mathrm{BC}$ \\
\hline 2 & $19.00 \mathrm{abc}$ & $19.33 \mathrm{ab}$ & 19.17 AB & $20.25 \mathrm{ab}$ & $20.50 \mathrm{ab}$ & 20.36 AB \\
\hline 3 & $19.33 \mathrm{ab}$ & $20.33 \mathrm{ab}$ & $19.83 \mathrm{AB}$ & $19.81 \mathrm{bc}$ & $21.50 \mathrm{a}$ & $20.65 \mathrm{AB}$ \\
\hline 4 & $16.67 \mathrm{bcd}$ & $17.33 \mathrm{bcd}$ & $17.00 \mathrm{~B}$ & $18.50 \mathrm{~b}$ & $19.50 \mathrm{bc}$ & 19.00 BC \\
\hline 5 & $17.33 \mathrm{bcd}$ & $18.67 \mathrm{abc}$ & 18.00 AB & $18.50 \mathrm{~b}$ & $18.50 \mathrm{~b}$ & $18.50 \mathrm{~B}$ \\
\hline 6 & $20.33 \mathrm{ab}$ & $20.67 \mathrm{ab}$ & $20.50 \mathrm{~A}$ & $21.00 \quad \mathrm{a}$ & $22.34 \mathrm{a}$ & $21.67 \mathrm{~A}$ \\
\hline 7 & $22.33 \mathrm{a}$ & $19.33 \mathrm{ab}$ & 20.83 A & $23.50 \mathrm{a}$ & $22.50 \mathrm{a}$ & $22.83 \mathrm{~A}$ \\
\hline 8 & $21.67 \mathrm{a}$ & 15.06 cde & $18.36 \mathrm{AB}$ & $21.00 \mathrm{a}$ & $21.34 \quad \mathrm{a}$ & $22.17 \mathrm{~A}$ \\
\hline Mean & $18.88 \mathrm{~A}$ & $17.80 \mathrm{~A}$ & & 20.11 A & 20.84 A & \\
\hline
\end{tabular}

Table2.Response of shoot length $(\mathrm{cm})$ of fruitful Washington navel orange trees to some bio and nano fertilizers (foliar spray) and two irrigation levels during 2016\&2017 experimental seasons.

\begin{tabular}{|c|c|c|c|c|c|c|}
\hline \multirow{3}{*}{ Treatments } & \multicolumn{6}{|c|}{ Shoot length (cm) } \\
\hline & Irrigation 1 & Irrigation 2 & Mean & Irrigation 1 & Irrigation 2 & Mean \\
\hline & \multicolumn{3}{|c|}{ First season } & \multicolumn{3}{|c|}{ Second season } \\
\hline 1 & $38.60 \mathrm{e}$ & $30.53 \mathrm{f}$ & 34.57 E & $33.54 \mathrm{c}$ & $33.00 \mathrm{c}$ & $33.27 \mathrm{C}$ \\
\hline 2 & $43.37 \mathrm{abc}$ & $41.57 \mathrm{~b}-\mathrm{e}$ & 42.47 BCD & $44.08 \mathrm{a}$ & $40.00 \mathrm{~b}$ & 42.04 B \\
\hline 3 & $44.10 \mathrm{abc}$ & $42.53 \mathrm{a}-\mathrm{d}$ & 43.32 ABC & $45.02 \mathrm{a}$ & $43.12 \mathrm{a}$ & 44.07 A \\
\hline 4 & 41.43 cde & $39.00 \mathrm{de}$ & $40.22 \quad D$ & $41.76 \mathrm{~b}$ & $41.10 \mathrm{~b}$ & $41.43 \mathrm{~B}$ \\
\hline 5 & 42.37 bcd & $39.30 \mathrm{de}$ & 40.83 CD & $42.20 \mathrm{~b}$ & $40.30 \mathrm{~b}$ & $41.25 \mathrm{~B}$ \\
\hline 6 & $45.10 \mathrm{ab}$ & $43.50 \mathrm{abc}$ & $44.30 \mathrm{AB}$ & $45.86 \mathrm{a}$ & $43.00 \mathrm{a}$ & 44.43 A \\
\hline 7 & $46.00 \mathrm{a}$ & $44.37 \mathrm{abc}$ & $45.18 \mathrm{~A}$ & $45.70 \mathrm{a}$ & $43.00 \mathrm{a}$ & $44.35 \mathrm{~A}$ \\
\hline 8 & $45.13 \mathrm{ab}$ & $43.80 \mathrm{abc}$ & 44.47 AB & $46.00 \mathrm{a}$ & $44.20 \mathrm{a}$ & 45.10 A \\
\hline Mean & $43.26 \mathrm{~A}$ & 40.58 B & & $43.27 \mathrm{~A}$ & $40.47 \quad$ B & \\
\hline
\end{tabular}


Table 3. Response of shoot diameter $(\mathrm{cm})$ of fruitful Washington navel orange trees to some bio and nano fertilizers (foliar spray) and two irrigation levels during 2016\&2017 experimental seasons.

\begin{tabular}{|c|c|c|c|c|c|c|}
\hline \multirow{3}{*}{ Treatments } & \multicolumn{6}{|c|}{ Shoot diameter $(\mathrm{cm})$} \\
\hline & Irrigation 1 & Irrigation2 & Mean & Irrigation1 & Irrigation2 & Mean \\
\hline & \multicolumn{3}{|c|}{ First season } & \multicolumn{3}{|c|}{ Second season } \\
\hline 1 & $0.240 \mathrm{ij}$ & $0.233 \mathrm{j}$ & $\begin{array}{ll}0.237 & F\end{array}$ & $0.255 \mathrm{c}$ & $0.245 \mathrm{c}$ & $0.250 \mathrm{D}$ \\
\hline 2 & $0.270 \mathrm{hij}$ & $0.290 \mathrm{ghi}$ & $0.280 \mathrm{E}$ & $0.320 \mathrm{~b}$ & $0.310 \mathrm{~b}$ & $0.310 \mathrm{CD}$ \\
\hline 3 & $0.337 \mathrm{~d}-\mathrm{g}$ & $0.317 \mathrm{fgh}$ & $0.327 \mathrm{D}$ & $0.340 \mathrm{~b}$ & $0.336 \mathrm{~b}$ & $0.338 \mathrm{BC}$ \\
\hline 4 & $0.367 \mathrm{~b}-\mathrm{f}$ & $0.333 \mathrm{efg}$ & $0.350 \mathrm{CD}$ & $0.360 \mathrm{~b}$ & $0.346 \mathrm{~b}$ & $0.352 \mathrm{BC}$ \\
\hline 5 & $0.373 \mathrm{~b}-\mathrm{e}$ & $0.353 \mathrm{c}-\mathrm{f}$ & 0.363 BCD & $0.390 \mathrm{~b}$ & $0.390 \mathrm{~b}$ & $0.390 \mathrm{AB}$ \\
\hline 6 & $0.373 \mathrm{~b}-\mathrm{e}$ & $0.357 \mathrm{c}-\mathrm{f}$ & $0.365 \mathrm{BC}$ & $0.410 \mathrm{a}$ & $0.396 \mathrm{a}$ & $0.403 \mathrm{~A}$ \\
\hline 7 & $0.450 \mathrm{a}$ & $0.417 \mathrm{ab}$ & $0.433 \mathrm{~A}$ & $0.450 \mathrm{a}$ & $0.450 \mathrm{a}$ & $0.450 \mathrm{~A}$ \\
\hline 8 & $0.407 \mathrm{abc}$ & $0.390 \mathrm{bcd}$ & 0.398 AB & $0.370 \mathrm{ab}$ & $0.350 \mathrm{ab}$ & $0.360 \mathrm{~B}$ \\
\hline Mean & $0.352 \mathrm{~A}$ & 0.336 A & & $0.362 \mathrm{~A}$ & $0.353 \mathrm{~A}$ & \\
\hline
\end{tabular}

Table 4. Response of No. of leaves / shoot of fruitful Washington navel orange trees to some bio and nano fertilizers (foliar spray) and two irrigation levels during 2016\&2017 experimental seasons.

\begin{tabular}{|c|c|c|c|c|c|c|c|}
\hline \multirow{3}{*}{ Treatments } & \multicolumn{7}{|c|}{ No. of leaves / shoot } \\
\hline & Irrigation 1 & Irrigation2 & Mean & Irrigation1 & Irrigation 2 & Mean & \\
\hline & \multicolumn{3}{|c|}{ First season } & \multicolumn{4}{|c|}{ Second season } \\
\hline 1 & $38.00 \mathrm{gh}$ & $37.33 \mathrm{~h}$ & $37.67 \quad \mathrm{~F}$ & $37.00 \mathrm{c}$ & $39.34 \mathrm{c}$ & 38.17 & $\mathbf{C}$ \\
\hline 2 & $40.00 \mathrm{e}-\mathrm{h}$ & $38.00 \mathrm{gh}$ & 39.00 EF & $40.67 \mathrm{~b}$ & $39.67 \mathrm{c}$ & 39.67 & $\mathbf{C}$ \\
\hline 3 & $41.67 \mathrm{def}$ & $38.33 \mathrm{gh}$ & $40.00 \mathrm{E}$ & $42.00 \mathrm{~b}$ & $44.00 \mathrm{a}$ & 43.00 & B \\
\hline 4 & $42.00 \mathrm{de}$ & $39.00 \mathrm{fgh}$ & 40.50 DE & $40.45 \mathrm{~b}$ & $40.21 \mathrm{~b}$ & 40.33 & $\mathbf{C}$ \\
\hline 5 & $44.33 \mathrm{bcd}$ & 40.67 efg & 42.50 CD & $45.30 \mathrm{a}$ & $41.36 \mathrm{~b}$ & 43.33 & B \\
\hline 6 & $45.67 \mathrm{ab}$ & 42.33 cde & 44.00 BC & $46.66 \mathrm{a}$ & $42.00 \mathrm{~b}$ & 44.33 & B \\
\hline 7 & $47.33 \mathrm{a}$ & $45.00 \mathrm{abc}$ & 46.17 A & $46.80 \mathrm{a}$ & $46.86 \mathrm{a}$ & 46.83 & $\mathbf{A}$ \\
\hline 8 & $46.67 \mathrm{ab}$ & $44.00 \mathrm{bcd}$ & $45.33 \mathrm{AB}$ & $46.00 \mathrm{a}$ & $44.00 \mathrm{a}$ & 45.00 & $\mathbf{A}$ \\
\hline Mean & $43.21 \mathrm{~A}$ & 40.58 B & & $45.62 \mathrm{~A}$ & 42.18 B & & \\
\hline
\end{tabular}

Table 5. Response of leaves fresh weight $(\mathrm{g})$ of fruitful Washington navel orange trees to some bio and nano fertilizers (foliar spray) and two irrigation levels during 2016\&2017 experimental seasons.

\begin{tabular}{|c|c|c|c|c|c|c|}
\hline \multirow{2}{*}{ Treatments } & \multicolumn{6}{|c|}{ leaves fresh weight ( g) } \\
\hline & Irrigation 1 & Irrigation2 & Mean & Irrigation 1 & Irrigation2 & Mean \\
\hline & \multicolumn{3}{|c|}{ First season } & \multicolumn{3}{|c|}{ Second season } \\
\hline 1 & $43.33 \mathrm{j}$ & $43.07 \mathrm{j}$ & $43.20 \mathrm{~F}$ & $43.54 \mathrm{f}$ & $43.58 \mathrm{f}$ & $43.56 \mathrm{~F}$ \\
\hline 2 & $67.76 \mathrm{de}$ & $54.20 \mathrm{gh}$ & $60.98 \mathrm{D}$ & $64.80 \mathrm{~d}$ & $62.70 \mathrm{~d}$ & $63.75 \mathrm{D}$ \\
\hline 3 & $72.70 \mathrm{bcd}$ & $61.61 \mathrm{f}$ & $67.15 \mathrm{C}$ & $70.98 \mathrm{c}$ & $70.85 \mathrm{c}$ & $71.30 \mathrm{C}$ \\
\hline 4 & $59.05 \mathrm{fg}$ & $47.05 \mathrm{ij}$ & 53.05 E & $55.09 \mathrm{e}$ & $55.10 \mathrm{e}$ & $55.08 \mathrm{E}$ \\
\hline 5 & 63.07 ef & $50.45 \mathrm{hi}$ & $56.76 \mathrm{E}$ & $59.12 \mathrm{e}$ & $59.24 \mathrm{e}$ & $59.18 \mathrm{E}$ \\
\hline 6 & $75.12 \mathrm{abc}$ & $69.59 \mathrm{~cd}$ & $72.35 \mathrm{~B}$ & $78.95 \mathrm{a}$ & $72.85 \mathrm{~b}$ & $75.90 \mathrm{~B}$ \\
\hline 7 & 80.84 a & $76.28 \mathrm{ab}$ & $78.56 \mathrm{~A}$ & $85.95 \mathrm{a}$ & $75.91 \mathrm{~b}$ & 80.93 A \\
\hline 8 & $78.54 \mathrm{ab}$ & $73.81 \mathrm{bc}$ & $76.17 \mathrm{AB}$ & 80.44 a & 78.69 a & 79.43 AB \\
\hline Mean & $67.55 \mathrm{~A}$ & 59.51 В & & $67.36 \mathrm{~A}$ & $64.87 \mathrm{~B}$ & \\
\hline
\end{tabular}


Table 6. Response of leaves dry weight ( $\mathrm{g}$ ) of fruitful Washington navel orange trees to some bio and nano fertilizers (foliar spray) and two irrigation levels during 2016\&2017 experimental seasons.

\begin{tabular}{|c|c|c|c|c|c|c|}
\hline \multirow{3}{*}{ Treatments } & \multicolumn{6}{|c|}{ Leaves dry weight ( g ) } \\
\hline & Irrigation 1 & Irrigation2 & Mean & Irrigation1 & Irrigation2 & Mean \\
\hline & \multicolumn{3}{|c|}{ First season } & \multicolumn{3}{|c|}{ Second season } \\
\hline 1 & 24.83 ef & $21.80 \mathrm{f}$ & $23.32 \mathrm{D}$ & $25.42 \mathrm{e}$ & $25.42 \mathrm{e}$ & $25.42 \mathrm{E}$ \\
\hline 2 & $32.73 \mathrm{bcd}$ & $23.80 \mathrm{ef}$ & 28.27 D & $29.78 \mathrm{~d}$ & $29.15 \mathrm{~d}$ & 29.27 DE \\
\hline 3 & $39.63 \mathrm{ab}$ & $28.83 \mathrm{de}$ & 34.23 BC & $34.13 \mathrm{c}$ & $30.60 \mathrm{~d}$ & 32.68 CD \\
\hline 4 & 35.30 a-d & $31.87 \mathrm{~cd}$ & $33.58 \mathrm{C}$ & $34.93 \mathrm{~b}$ & $32.30 \mathrm{c}$ & $33.93 \mathrm{C}$ \\
\hline 5 & $36.20 \mathrm{abc}$ & $37.90 \mathrm{abc}$ & $37.05 \mathrm{ABC}$ & $35.30 \mathrm{~b}$ & $35.85 \mathrm{~b}$ & $35.65 \mathrm{BC}$ \\
\hline 6 & $37.50 \mathrm{abc}$ & $37.00 \mathrm{abc}$ & 37.25 ABC & $40.00 \mathrm{a}$ & $36.30 \mathrm{~b}$ & 38.47 AB \\
\hline 7 & $40.07 \mathrm{a}$ & $38.97 \mathrm{ab}$ & $39.52 \mathrm{~A}$ & $41.30 \mathrm{a}$ & $39.65 \mathrm{a}$ & 40.85 A \\
\hline 8 & $39.53 \mathrm{ab}$ & $38.23 \mathrm{abc}$ & 38.88 AB & 40.40 a & $39.55 \mathrm{a}$ & 39.25 AB \\
\hline Mean & $35.72 \mathrm{~A}$ & 32.30 B & & $31.41 \mathrm{~A}$ & 29.82 B & \\
\hline
\end{tabular}

Table 7. Response of nitrogen percentage (N\%) of fruitful Washington navel orange trees to some bio and nano fertilizers (foliar spray) and two irrigation levels during 2016\&2017 experimental seasons.

\begin{tabular}{|c|c|c|c|c|c|c|}
\hline \multirow{3}{*}{ Treatments } & \multicolumn{6}{|c|}{$\mathbf{N} \%$} \\
\hline & Irrigation 1 & Irrigation2 & Mean & Irrigation1 & Irrigation2 & Mean \\
\hline & \multicolumn{3}{|c|}{ First season } & \multicolumn{3}{|c|}{ Second season } \\
\hline 1 & $1.873 \mathrm{c}$ & $1.650 \mathrm{c}$ & $1.762 \mathrm{D}$ & $1.860 \mathrm{~d}$ & $1.866 \mathrm{~d}$ & $1.863 \mathrm{D}$ \\
\hline 2 & $2.823 \mathrm{ab}$ & $2.753 \mathrm{~b}$ & $2.788 \mathrm{BC}$ & $2.650 \mathrm{c}$ & $2.670 \mathrm{c}$ & $2.660 \mathrm{C}$ \\
\hline 3 & $2.880 \mathrm{ab}$ & $2.783 \mathrm{ab}$ & $2.832 \mathrm{BC}$ & $2.880 \mathrm{~b}$ & $2.904 \mathrm{~b}$ & 2.892 B \\
\hline 4 & $2.760 \mathrm{~b}$ & $2.633 \mathrm{~b}$ & $2.697 \mathrm{C}$ & $2.740 \mathrm{c}$ & $3.734 \mathrm{c}$ & 2.737 BC \\
\hline 5 & $2.790 \mathrm{ab}$ & $2.717 \mathrm{~b}$ & $2.753 \mathrm{C}$ & $2.610 \mathrm{c}$ & $2.614 \mathrm{c}$ & $2.612 \mathrm{C}$ \\
\hline 6 & $3.103 \mathrm{ab}$ & $2.927 \mathrm{ab}$ & 3.015ABC & $3.470 \mathrm{~b}$ & $3.494 \mathrm{a}$ & $3.482 \mathrm{~A}$ \\
\hline 7 & $3.500 \mathrm{a}$ & $3.330 \mathrm{ab}$ & $3.415 \mathrm{~A}$ & $3.480 \mathrm{a}$ & $3.460 \mathrm{a}$ & $3.470 \mathrm{~A}$ \\
\hline 8 & $3.310 \mathrm{ab}$ & $3.237 \mathrm{ab}$ & $3.273 \mathrm{AB}$ & $3.390 \mathrm{a}$ & $3.330 \mathrm{a}$ & $3.360 \mathrm{~A}$ \\
\hline Mean & $2.880 \mathrm{~A}$ & $2.754 \mathrm{~A}$ & & $2.885 \mathrm{~A}$ & $2.762 \mathrm{~A}$ & \\
\hline
\end{tabular}

Table 8. Response of phosphorus percentage (P\%) of fruitful Washington navel orange trees to some bio and nano fertilizers (foliar spray) and two irrigation levels during $2016 \& 2017$ experimental seasons.

\begin{tabular}{|c|c|c|c|c|c|c|}
\hline \multirow{3}{*}{ Treatments } & \multicolumn{6}{|c|}{ P \% } \\
\hline & Irrigation 1 & Irrigation2 & Mean & Irrigation 1 & Irrigation 2 & Mean \\
\hline & \multicolumn{3}{|c|}{ First season } & \multicolumn{3}{|c|}{ Second season } \\
\hline 1 & $0.289 \mathrm{de}$ & $0.144 \mathrm{e}$ & $0.217 \mathrm{D}$ & $0.272 \mathrm{c}$ & $0.248 \mathrm{c}$ & $0.260 \mathrm{C}$ \\
\hline 2 & $0.508 \mathrm{bc}$ & $0.389 \mathrm{~cd}$ & 0.449 ABC & $0.450 \mathrm{~b}$ & $0.448 \mathrm{~b}$ & 0.449 AB \\
\hline 3 & $0.650 \mathrm{ab}$ & $0.388 \mathrm{~cd}$ & 0.519 AB & $0.530 \mathrm{a}$ & $0.516 \mathrm{a}$ & $0.523 A$ \\
\hline 4 & $0.486 \mathrm{bc}$ & $0.291 \mathrm{de}$ & $0.388 \mathrm{C}$ & $0.370 \mathrm{e}$ & $0.362 \mathrm{c}$ & $0.366 \mathrm{C}$ \\
\hline 5 & $0.499 \mathrm{bc}$ & $0.334 \mathrm{~cd}$ & $0.417 \mathrm{BC}$ & $0.450 \mathrm{~b}$ & $0.418 \quad b$ & 0.434 B \\
\hline 6 & $0.650 \mathrm{ab}$ & $0.401 \mathrm{~cd}$ & $0.525 \mathrm{AB}$ & $0.550 \mathrm{a}$ & $0.520 \mathrm{a}$ & $0.535 \mathrm{~A}$ \\
\hline 7 & $0.687 \mathrm{a}$ & $0.419 \mathrm{~cd}$ & $0.553 \mathrm{~A}$ & $0.590 \mathrm{a}$ & $0.588 \mathrm{a}$ & 0.589 A \\
\hline 8 & $0.657 \mathrm{ab}$ & $0.429 \mathrm{~cd}$ & $0.543 \mathrm{~A}$ & $0.580 \quad \mathrm{a}$ & $0.570 \quad \mathrm{a}$ & $0.575 \mathrm{~A}$ \\
\hline Mean & 0.553 A & 0.349 B & & $0.474 \mathrm{~A}$ & 0.459 B & \\
\hline
\end{tabular}


Table 9. Response of potassium percentage (K\%) of fruitful Washington navel orange trees to some bio and nano fertilizers (foliar spray) and two irrigation levels during 2016\&2017 experimental seasons.

\begin{tabular}{cccccccc}
\hline \multirow{2}{*}{ Treatments } & \multicolumn{7}{c}{ K \% } \\
\cline { 2 - 7 } & Irrigation 1 & Irrigation2 & Mean & Irrigation 1 & Irrigation2 & Mean \\
\cline { 2 - 8 } & $1.081 \mathrm{a}$ & First season & $1.518 \mathrm{a}$ & $\mathbf{1 . 3 0 0} \mathbf{A}$ & $1.072 \mathrm{a}$ & $1.076 \mathrm{a}$ & $\mathbf{1 . 0 7 4} \mathbf{A}$ \\
\hline $\mathbf{1}$ & $1.610 \mathrm{a}$ & $1.528 \mathrm{a}$ & $\mathbf{1 . 5 6 9} \mathbf{A}$ & $1.595 \mathrm{a}$ & $1.583 \mathrm{a}$ & $\mathbf{1 . 5 8 9} \mathbf{A}$ \\
$\mathbf{2}$ & $1.689 \mathrm{a}$ & $1.687 \mathrm{a}$ & $\mathbf{1 . 6 8 8} \mathbf{A}$ & $1.720 \mathrm{a}$ & $1.717 \mathrm{a}$ & $\mathbf{1 . 7 1 4} \mathbf{A}$ \\
$\mathbf{3}$ & $1.377 \mathrm{a}$ & $1.364 \mathrm{a}$ & $\mathbf{1 . 3 7 0} \mathbf{A}$ & $1.501 \mathrm{a}$ & $1.509 \mathrm{a}$ & $\mathbf{1 . 5 0 5} \mathbf{A}$ \\
$\mathbf{4}$ & $1.471 \mathrm{a}$ & $1.414 \mathrm{a}$ & $\mathbf{1 . 4 4 2} \mathbf{A}$ & $1.571 \mathrm{a}$ & $1.559 \mathrm{a}$ & $\mathbf{1 . 5 6 5} \mathbf{A}$ \\
$\mathbf{5}$ & $1.730 \mathrm{a}$ & $1.717 \mathrm{a}$ & $\mathbf{1 . 7 2 4} \mathbf{A}$ & $1.732 \mathrm{a}$ & $1.742 \mathrm{a}$ & $\mathbf{1 . 7 3 6} \mathbf{A}$ \\
$\mathbf{6}$ & $1.807 \mathrm{a}$ & $1.746 \mathrm{a}$ & $\mathbf{1 . 7 7 6} \mathbf{A}$ & $1.989 \mathrm{a}$ & $1.983 \mathrm{a}$ & $\mathbf{1 . 9 8 6} \mathbf{A}$ \\
$\mathbf{7}$ & $1.760 \mathrm{a}$ & $1.720 \mathrm{a}$ & $\mathbf{1 . 7 4 0} \mathbf{A}$ & $1.772 \mathrm{a}$ & $1.756 \mathrm{a}$ & $\mathbf{1 . 7 6 4} \mathbf{A}$ \\
$\mathbf{8}$ & $\mathbf{1 . 5 6 6} \mathbf{A}$ & $\mathbf{1 . 5 8 7} \mathbf{A}$ & $\mathbf{1 . 6 1 9} \mathbf{A}$ & $\mathbf{1 . 6 1 7} \mathbf{A}$ & \\
\hline Mean & & & & &
\end{tabular}

Table 10. Response of calcium percentage $(\mathrm{Ca} \%)$ of fruitful Washington navel orange trees to some bio and nano fertilizers (foliar spray) and two irrigation levels during 2016\&2017 experimental seasons.

\begin{tabular}{|c|c|c|c|c|c|c|}
\hline \multirow{3}{*}{ Treatments } & \multicolumn{6}{|c|}{$\mathrm{Ca} \%$} \\
\hline & Irrigation 1 & Irrigation 2 & Mean & Irrigation 1 & Irrigation 2 & Mean \\
\hline & \multicolumn{3}{|c|}{ First season } & \multicolumn{3}{|c|}{ Second season } \\
\hline 1 & 3.944 e-h & $3.272 \mathrm{~h}$ & $3.608 \mathrm{D}$ & $3.690 \mathrm{c}$ & $3.706 \mathrm{c}$ & $3.698 \mathrm{C}$ \\
\hline 2 & 4.453 b-f & $3.596 \mathrm{gh}$ & 4.024 CD & $4.170 \mathrm{~b}$ & $4.168 \mathrm{~b}$ & 4.169 BC \\
\hline 3 & 4.617 a-e & $3.764 \mathrm{fgh}$ & 4.190 BC & $4.351 \mathrm{~b}$ & $4.319 \mathrm{~b}$ & 4.330 BC \\
\hline 4 & $4.921 \mathrm{a}-\mathrm{d}$ & $4.033 \mathrm{efg}$ & 4.477 BC & $4.630 \mathrm{ab}$ & $4.600 \mathrm{ab}$ & $4.615 \mathrm{AB}$ \\
\hline 5 & $5.029 \mathrm{abc}$ & 4.171 efg & 4.600 $\mathrm{AB}$ & $4.451 \mathrm{ab}$ & $4.457 \mathrm{ab}$ & 4.654 AB \\
\hline 6 & $5.073 \mathrm{ab}$ & $4.296 \mathrm{~d}-\mathrm{g}$ & 4.684 AB & $4.720 \mathrm{ab}$ & $4.717 \mathrm{ab}$ & $4.717 \mathrm{AB}$ \\
\hline 7 & $5.262 \mathrm{a}$ & 4.618 a-e & $4.940 \mathrm{~A}$ & $5.316 \mathrm{a}$ & $5.300 \mathrm{a}$ & $5.308 \mathrm{~A}$ \\
\hline 8 & $5.173 \mathrm{a}$ & $4.333 \quad \mathrm{c}-\mathrm{f}$ & $4.753 \mathrm{~A}$ & $4.872 \mathrm{ab}$ & $4.882 \mathrm{ab}$ & $4.877 \mathrm{AB}$ \\
\hline Mean & $4.809 \mathrm{~A}$ & $4.010 \quad B$ & & $4.525 \mathrm{~A}$ & $4.159 \quad B$ & \\
\hline
\end{tabular}

Table11. Response of magnesium percentage $(\mathrm{Mg} \%)$ of fruitful Washington navel orange trees to some bio and nano fertilizers (foliar spray) and two irrigation levels during 2016\&2017 experimental seasons.

\begin{tabular}{|c|c|c|c|c|c|c|}
\hline \multirow{3}{*}{ Treatments } & \multicolumn{6}{|c|}{$\operatorname{Mg} \%$} \\
\hline & Irrigation 1 & Irrigation2 & Mean & Irrigation1 & Irrigation 2 & Mean \\
\hline & \multicolumn{3}{|c|}{ First season } & \multicolumn{3}{|c|}{ Second season } \\
\hline 1 & $0.237 \mathrm{hi}$ & $0.233 \quad \mathrm{i}$ & $0.235 \quad E$ & $0.250 \mathrm{c}$ & $0.230 \mathrm{c}$ & $0.240 \quad E$ \\
\hline 2 & 0.469 def & $0.342 \mathrm{gh}$ & 0.405 D & $0.430 \mathrm{~b}$ & $0.406 \mathrm{~b}$ & 0.418 B \\
\hline 3 & 0.482 cde & $0.366 \mathrm{fg}$ & $0.424 \mathrm{D}$ & $0.450 \mathrm{a}$ & $0.420 \mathrm{a}$ & 0.435 B \\
\hline 4 & 0.482 cde & 0.405 efg & $0.444 \mathrm{CD}$ & $0.450 \mathrm{~b}$ & $0.436 \mathrm{~b}$ & 0.443 B \\
\hline 5 & $0.508 \mathrm{~b}-\mathrm{e}$ & $0.423 \mathrm{~d}-\mathrm{g}$ & $0.465 B C D$ & $0.520 \mathrm{~b}$ & $0.495 \mathrm{~b}$ & 0.505 B \\
\hline 6 & $0.603 \mathrm{ab}$ & $0.459 \mathrm{def}$ & $0.531 \mathrm{AB}$ & $0.540 \mathrm{~b}$ & $0.532 \mathrm{~b}$ & $0.536 B$ \\
\hline 7 & $0.580 \mathrm{abc}$ & $0.455 \mathrm{def}$ & $0.517 \mathrm{ABC}$ & $0.650 \mathrm{a}$ & $0.626 \mathrm{a}$ & 0.638 A \\
\hline 8 & $0.621 \mathrm{a}$ & $0.522 \mathrm{a}-\mathrm{d}$ & $0.572 \mathrm{~A}$ & $0.620 \mathrm{a}$ & $0.606 \mathrm{a}$ & $0.613 \mathrm{~A}$ \\
\hline Mean & 0.498 A & 0.401 B & & 0.489 A & 0.469 B & \\
\hline
\end{tabular}


Table 12. Response of iron at ppm (Fe) of fruitful Washington navel orange trees to some bio and nano fertilizers (foliar spray) and two irrigation levels during 2016\&2017 experimental seasons.

\begin{tabular}{|c|c|c|c|c|c|c|}
\hline \multirow{2}{*}{ Treatments } & \multicolumn{6}{|c|}{ Fe (ppm) } \\
\hline & Irrigation1 & Irrigation2 & Mean & Irrigation 1 & Irrigation2 & Mean \\
\hline & \multicolumn{3}{|c|}{ First season } & \multicolumn{3}{|c|}{ Second season } \\
\hline 1 & $82.00 \mathrm{f}$ & $79.67 \mathrm{f}$ & 80.83 E & $90.40 \mathrm{e}$ & $72.60 \mathrm{e}$ & 81.50 D \\
\hline 2 & $111.3 \mathrm{~cd}$ & $98.00 \mathrm{e}$ & 104.7 D & $115.80 \mathrm{c}$ & $101.90 \mathrm{~d}$ & $108.7 \mathrm{C}$ \\
\hline 3 & $117.7 \mathrm{abc}$ & $105.7 \mathrm{de}$ & $111.7 \mathrm{C}$ & $115.40 \mathrm{c}$ & $117.00 \mathrm{bc}$ & $116.2 \mathrm{BC}$ \\
\hline 4 & $119.3 \mathrm{abc}$ & $115.0 \mathrm{bcd}$ & 117.2 BC & $114.90 \mathrm{c}$ & $122.50 \mathrm{~b}$ & $118.7 \mathrm{AB}$ \\
\hline 5 & $121.0 \mathrm{ab}$ & $119.7 \mathrm{abc}$ & $120.3 \mathrm{AB}$ & $124.00 \mathrm{ab}$ & $120.00 \mathrm{~b}$ & $122.0 \mathrm{AB}$ \\
\hline 6 & $124.0 \mathrm{ab}$ & $125.3 \mathrm{a}$ & $124.7 \mathrm{~A}$ & $125.50 \mathrm{ab}$ & $117.50 \mathrm{~b}$ & 123.0 AB \\
\hline 7 & $125.3 \mathrm{a}$ & $124.3 \mathrm{ab}$ & $124.8 \mathrm{~A}$ & $130.00 \mathrm{a}$ & $122.00 \mathrm{~b}$ & $126.0 \mathrm{~A}$ \\
\hline 8 & $124.7 \mathrm{a}$ & $122.7 \mathrm{ab}$ & $123.7 \mathrm{AB}$ & $128.30 \quad \mathrm{a}$ & $120.30 \mathrm{~b}$ & 124.3 AB \\
\hline Mean & $115.7 \mathrm{~A}$ & $111.3 \mathrm{~B}$ & & 118.04A & $111.73 \mathrm{~B}$ & \\
\hline
\end{tabular}

Table 13. Response of manganese at ppm (Mn) of fruitful Washington navel orange trees to some bio and nano fertilizers (foliar spray) and two irrigation levels during 2016\&2017 experimental seasons.

\begin{tabular}{|c|c|c|c|c|c|c|}
\hline \multirow{3}{*}{ Treatments } & \multicolumn{6}{|c|}{ Mn (ppm) } \\
\hline & Irrigation1 & Irrigation 2 & Mean & Irrigation 1 & Irrigation2 & Mean \\
\hline & \multicolumn{3}{|c|}{ First season } & \multicolumn{3}{|c|}{ Second season } \\
\hline 1 & $38.67 \mathrm{i}$ & $37.00 \mathrm{i}$ & $37.83 \mathrm{~F}$ & 39.21 & $39.45 \mathrm{f}$ & 39.33 F \\
\hline 2 & $76.00 \mathrm{de}$ & $59.93 \mathrm{gh}$ & 67.97 D & $71.81 \mathrm{bc}$ & $69.75 \mathrm{bc}$ & 70.78 CD \\
\hline 3 & $85.03 \mathrm{bc}$ & $64.77 \mathrm{fg}$ & $74.90 \mathrm{C}$ & $79.88 \mathrm{ab}$ & $73.66 \mathrm{ac}$ & 76.72 BC \\
\hline 4 & 69.63 ef & $54.63 \mathrm{~h}$ & $62.13 \mathrm{E}$ & $65.90 \mathrm{~cd}$ & 59.94 e & $62.92 \mathrm{E}$ \\
\hline 5 & 73.47 de & $56.10 \mathrm{~h}$ & 64.78 DE & $70.60 \mathrm{bc}$ & $60.60 \mathrm{~d}$ & 65.60 DE \\
\hline 6 & $88.70 \mathrm{ab}$ & 68.27 ef & $78.48 \mathrm{BC}$ & $81.34 \mathrm{a}$ & $79.40 \mathrm{ab}$ & 80.37 AB \\
\hline 7 & $94.40 \mathrm{a}$ & $79.50 \mathrm{~cd}$ & 86.95 A & $90.00 \mathrm{a}$ & $86.26 \mathrm{a}$ & 87.13 A \\
\hline 8 & $93.30 \mathrm{a}$ & $73.43 \mathrm{de}$ & 83.37 AB & $89.80 \mathrm{a}$ & $81.70 \mathrm{a}$ & $85.75 \mathrm{~A}$ \\
\hline Mean & $77.40 \mathrm{~A}$ & $61.70 \mathrm{~B}$ & & $73.57 \mathrm{~A}$ & 68.85 B & \\
\hline
\end{tabular}

Table 14.Response of zinc at ppm $(\mathrm{Zn})$ of fruitful Washington navel orange trees to some bio and nano fertilizers (foliar spray) and two irrigation levels during 2016\&2017 experimental seasons.

\begin{tabular}{|c|c|c|c|c|c|c|}
\hline \multirow{2}{*}{ Treatments } & \multicolumn{6}{|c|}{ Zn (ppm) } \\
\hline & Irrigation1 & Irrigation 2 & Mean & Irrigation1 & Irrigation2 & Mean \\
\hline & \multicolumn{3}{|c|}{ First season } & \multicolumn{3}{|c|}{ Second season } \\
\hline 1 & $65.30 \mathrm{ef}$ & $64.80 \mathrm{f}$ & $65.05 \quad \mathrm{E}$ & $70.30 \mathrm{c}$ & $60.44 \mathrm{~d}$ & $\begin{array}{ll}65.37 & D\end{array}$ \\
\hline 2 & $90.90 \mathrm{a}$ & $73.10 \mathrm{de}$ & 82.00 C & $82.54 \mathrm{~b}$ & $80.74 \mathrm{~b}$ & 81.64 BC \\
\hline 3 & $90.00 \mathrm{ab}$ & $75.43 \mathrm{~cd}$ & $82.72 \mathrm{C}$ & $85.51 \mathrm{ab}$ & $81.31 \mathrm{~b}$ & 83.41 AB \\
\hline 4 & 82.07 bc & 67.14 ef & 74.61 D & $80.58 \mathrm{~b}$ & $72.42 \mathrm{c}$ & 76.50 C \\
\hline 5 & $88.33 \mathrm{ab}$ & $72.09 \mathrm{def}$ & 80.21 CD & $80.40 \mathrm{~b}$ & $78.54 \mathrm{bc}$ & 79.47 C \\
\hline 6 & $92.00 \mathrm{a}$ & $77.40 \mathrm{~cd}$ & 84.70 BC & $90.00 \mathrm{a}$ & $78.08 \mathrm{ab}$ & 84.04 AB \\
\hline 7 & $93.10 \mathrm{a}$ & $88.02 \mathrm{ab}$ & $90.56 \mathrm{~A}$ & $96.02 \mathrm{a}$ & $90.02 \mathrm{a}$ & 93.02 A \\
\hline 8 & $92.10 \mathrm{a}$ & $85.97 \mathrm{ab}$ & 89.03 AB & $92.65 \mathrm{a}$ & 88.61 a & $90.63 \mathrm{~A}$ \\
\hline Mean & $86.73 \mathrm{~A}$ & $75.49 \quad$ B & & $84.75 \mathrm{~A}$ & 78.77 B & \\
\hline
\end{tabular}

Values within the same column and raw for any of two investigated factors were individually differentiated by capital letters, while for the interaction small letters were used, mean followed by the same letter/s were not significantly different at $5 \%$ level.

1-Control(watersprayoftreessubjectedtoonlythe N, P, K fertilizersprogramsadoptedinthefarm).

2-Foliarspraywithyeast extract at $150 \mathrm{ml} /$ liter.

3-Foliar spraywithyeast extract at $200 \mathrm{ml} / \mathrm{liter}$.

4-Foliarspraywithlithovit at $2.0 \mathrm{~g} / \mathrm{liter}$.

5-Foliarspraywithlithovit at $4.0 \mathrm{~g} / \mathrm{liter}$.

6-Foliarspraywithyeast extract at $150 \mathrm{ml} / \mathrm{liter}+$ lithovit at $2.0 \mathrm{~g} / \mathrm{liter}$.

7-Foliarspraywithyeast extract at $150 \mathrm{ml} / \mathrm{liter}+$ lithovit at $4.0 \mathrm{~g} / \mathrm{liter}$.

8-Foliarspraywithyeast extract at $200 \mathrm{ml} / \mathrm{liter}+$ lithovit at $4.0 \mathrm{~g} / \mathrm{liter}$.

Irrigation $1=(3894.2 \mathrm{~m} 3 /$ year $) \quad$ Irrigation $2=(3127.8 \mathrm{~m} 3 /$ year $)$ 


\section{References}

Abd El-Aal, M. M. and Rania- Eid, S. M. (2018): Effect of foliar spray with lithovit and amino acids on growth, bio constituents, anatomical and yield features of soybean plant. 4th International Conference on Biotechnology Applications in Agriculture (ICBAA), Benha University, Moshtohor and Hurghada , 4-7 April 2018 , Egypt . Plant Biotechnology, 187-201.

Abd El ghafar, M. S.; M. T. Al-Abd; A. A. Helaly and A. M. Rashwan (2016):Foliar application of lithovit and rose Water as factor for increasing onion seed production, Nat. Sci. 14(3): $53-61$.

Abo Sedera, F. A.; N. S. Shafshak; A. S. Shams; M. A. Abuo-Soud and M. H. Mohammed (2015): The utilize of vermicomposting outputs in substrate culture for producing snap bean. Annals Agric. Sci., Moshtohor, 53(2):139-151.

Ahmed, F.F.; A.E.M. Mansour; M.A.A. Montasser; M.A. Merwad and E.A.M. Mostafa (2013): Response of Valencia orange trees to foliar application of roselle, turmeric and seaweed extracts. J. Appl. Sci. Res., 9(1): 960-964.

Amer, S.S.A., (2004). Growth, green pods yield and seeds yield of common bean (Phaseolus vulgaris L) as affected by active dry yeast, salicylic acid and their interaction. J. Agric. Sci. Mansoura. Univ., 29(3): 1407- 1422.

Ayed, M.E., (2016). Physiological Studies on Washington navel orange trees grown in new reclaimed soils. M.Sc. Thesis, Faculty of Agric, Moshtohor, Benha University.

Badawy-Sabah, M. (2005). Histophysiological effects of yeast and kinetin application on fruit characteristics and fruit growth stages of Balady mandarin cultivar. Ph. D. Thesis Hort. Pomology Fac. of Agric, Assuit University Egypt.

Bakry, KH. A. (2007): Response of Jaffa orange cultivar to spray with yeast extract and promalin. Egyptian J. of app. Sci., 22(10A) : PP. 195-210.

Bilal, B.A. (2010): Lithovit: An innovative fertilizer. The 3rd e Conference on Agricultural Biosciences (IeCAB 2010), 1st- 15th June 2010. http://www.slideserve.com/madison/lithovitan-

Brown, J. D. and Lilliand, O. (1946).Rapid determination of potassium and sodium in plant material and soil extract by flam photometer. Proc. Amer. Soc. Hort. Sci., 48: 341-346.

Brown, L. R. (1999). Feeding nine billions. In L. Storke (ed. State of the world).

Carmen, B.; R. Sumalan; S. Gadea and S. Vatca (2014): Physiological indicators study involved in productivity increasing in tomato. Proenvironment, 7: 218 - 224.

Cathoun, F. G. (1975). Influence of particle size and organic matter on water tension in selected Florida soils. Proc. Soil and Crop Sci. of Florida, 32: 111-113.
Chapman, H. D. and P. F. Pratt (1975): Methods of Analysis for Soils, Plant and Waters. Univ. of California. Division of Agric. Sci. $7^{\text {th }}$ Ed. P. 172173.

Crouch, I.J. andJ.VanStaden (1993): Commercial Sea weeds products as bio stimulants in horticulture. Home and Consumer Horti.1:19-76.

Demir, D.; Günes, A.; Inal, A. and Alpaslan, M. (2004). Effects of humic acids on the yield and mineral nutrition of cucumber (cucumissativus L.) grown with different salinity levels. Acta Horticulture, 492.

Duncan, B. D. (1955): Duncan`s multiple range and multiple F tests. Biometrics, 11: 1-42.

El-Shazly, S. M. andMoustafa, N. S. (2013). Enhancement yield, fruit quality and nutritional status of Washington navel orange trees by application ofbiostimulant. Journal of Applied Sciences Research;. 9(8):5030-5034.

Fareg, A. A. (2007): Evaluation of water flow measurement methods appropriate for on-farm management in Egypt. M. Sc. Thesis, Agric. Mechanization Dep. Fac, of Agric. Benha Univ.

Fletcher, R. A.; A. Gilley; N. Sankhla and T. D. Davis (1988): Triazoles as plant growth regulators and stress protectants. Horticultural Reviews 24: 55-138.

Ghatas Y.A. and Mohamed Y.F. (2018): Influence of Mineral, Micro-nutrients and Lithovit on Growth, Oil Productivity and Volatile Oil Constituents of CymbopogoncitrutsL. Plants. Middle East J. Agric. Res., 7(1): 162-174, 2018

Ismail, A. F.; Hussien, S. M.; El- Shall, S. A. and Fathi, M. A. (2007).Effect of irrigation and humic acid on Le-Conte pear trees. J. Agric. Sci., Mansoura Univ., 32(9): 7589-7603.

Kandil, E.A. and S. El-Feky (2006). Effect of soil matric potential on "Canino" apricot trees in sandy soil under drip irrigation. J. Agric. Sci. Mansoura Univ., 31 (9): 5867-5880.

Kumar, P. (2011): Nanotechnology in agriculture. Financing Agriculture, 34: 8-10.

Levin, I.; R. Assaf and B. A. Bravdo (1980). Irrigation water status and nutrient uptake in an apple orchard. Butterworths, Borough Green UK p. 230 .

Lu, C. M.; C. Y. Zhang; J. Q. Wen; G. R. Wu and M. X. Tao (2002): Research of the effect of nanometer materials on germination and growth enhancement of Glycine max and its mechanism. Soya Bean Science 21: 168-172.

Marschner, H. (1995): Mineral nutrition of higher plants. $2^{\text {nd }}$ ed. Acadimic press. Pub. New York (USA).

Maurer, M. A.; F. S. Davies and D. A. Craetz (1995). Reclaimed waste water irrigation and fertilization of mature "Redblush" grape fruit trees on Spodosols in Florida. J. Amer. Soc. Hort. Sci. 120 (3): 394-402, USA. (Hort. Abst. 65 No. 8: 7425). 
Ministry of Agriculture \& Land Reclamation; Economic Affairs Sector, Egypt 2017.

Nadi, E.; A. Aynehband and M. Mojaddam (2013): Effect of nano-iron chelate fertilizer on grain yield, protein percent and chlorophyll content of Faba bean (ViciafabaL.). Inter. J. of Biosci ., 3 (9): 267-272.

Nel, A.; T. Xia; L. Madler and N. Li (2006): Toxic potential of materials at the Nano level. Science 311: 622-627.

Nelson W.R. and Van Staden, J. (1984). The effect of seaweed concentrate on wheat culms. J. Plant Physiol. 115:433-437.

Piper, C. S. (1958): Soil and Plant Analysis. Inter. Sci. Publishers. New York, 213-217.

Pregl, F. (1945): Quantitive organic micro-analysis. $4^{\text {th }} \mathrm{Ed}$, and A. Churchill. LTD. London.

Saeed, W. T.; A. M. Abou el khashab and S. A. Aboutaleb (2005): Physiological studies on jojoba plants. B- Effect of some ecology stress on jojoba seedlings. Bulletin of Fac, of Agric, Cairo Univ., 56: 121-142.

Salisbury, F. B. and C.W. Ross (1985). Plant physiology. Wadsworth Pub. Co., Calif. USA p. 185 .
Sanchez, M. J.; A. Torrecillos and F. Del-Amor (1989). Effect of irrigation regimes on the stage of reproduction in Citrus limon L. cv. Verna. Advances in Horticultural Sciences, 3: 13-16.

Smirnoff, N. (1995). Antioxidant systems and plant response to the environment. In N. Smirnoff (Ed.), Environment and plant metabolism: Flexibility and acclimation (pp. 217-243). Oxford, UK: BIOS Scientific Publishers Ltd.

Snedecor, G. W. and W. G. Cochran (1980). Statistical Methods. Oxford and J.B.H. publishing com. $7^{\text {th }}$ edition, pp 593.

Wright, J. L. and J. C. Stark (1990). Irrigation of agricultural crops. Amer. Soc. Agron., 30: 112117.

Youssef, A. M. (1990). Physiological and morphological studies on some new citrus rootstocks. Ph.D. Thesis, Hort. Dept., Fac. Agric., Al-Azhar Univ., Cairo.

Zekri, M. (1991): Effects of PEG- induced water stress on two citrus cultivars. Journal of Plant Nutrition, 14 (1): 59-74.

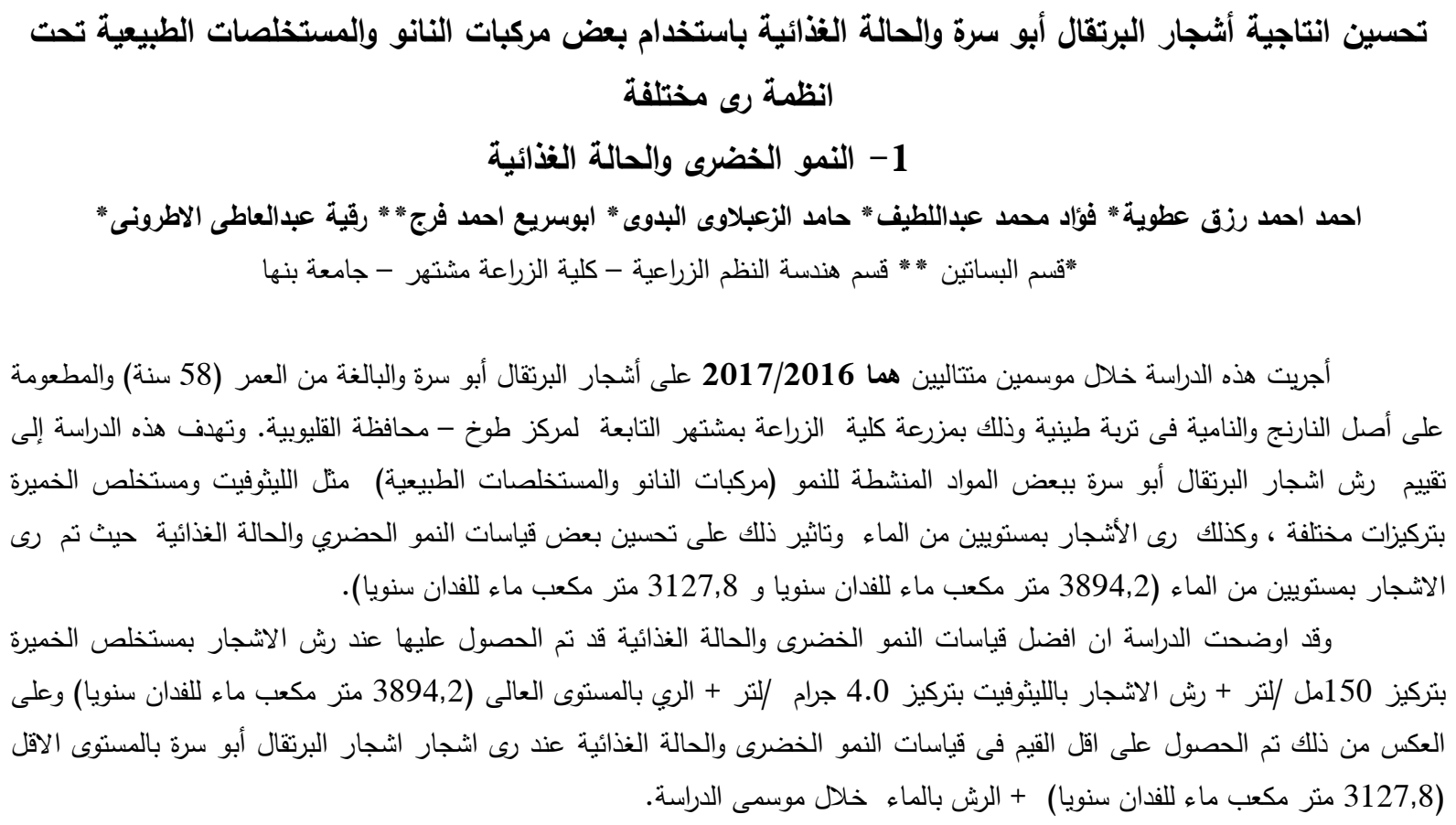

